\title{
A class of diagonal quasi-newton methods for large-scale convex minimization
}

\begin{abstract}
We study the convergence properties of a class of low memory methods for solving largescale unconstrained problems. This class of methods belongs to that of quasi-Newton family, except for which the approximation to Hessian, at each step, is updated by means of a diagonal matrix. Using appropriate scaling, we show that the methods can be implemented so as to be globally and $\backslash(\mathrm{R} \backslash)$-linearly convergent with standard inexact line searches. Preliminary numerical results suggest that the methods are good alternative to other low memory methods such as the CG and spectral gradient methods.
\end{abstract}

Keyword: Large-scale convex minimization; Quasi-Newton methods; Diagonal updating; Scaling; Globally and R-linearly convergent 